\title{
SELEÇÃO DE VARIÁVEIS E CONTROLE DE COMPOSIÇÃO POR INFERÊNCIA DE TEMPERATURA EM PROCESSO PRODUTIVO DO ETILBENZENO
}

\author{
P. R. A. MARIZ1 ${ }^{1}$ E. F. L. VIEIRA, W. Y. A. BRANDÃO ${ }^{1}$, L. O. A. ROJAS ${ }^{1}$, A. A. M. \\ $\mathrm{JUNIOR}^{1}$ \\ ${ }^{1}$ Universidade Federal da Paraíba, Departamento de Engenharia Química \\ E-mail para contato: rom3romariz@gmail.com
}

\begin{abstract}
RESUMO - Processos com múltiplas variáveis de entrada e múltiplas saídas (MIMO) são relativamente complexos quanto à definição do controle adequado, haja vista as interações decorrentes do acoplamento entre essas variáveis. Há, portanto, a necessidade de estabelecer uma estrutura de controle adequada e que forneça bom desempenho. Em busca dos melhores pares de variáveis Manipuladas-Controladas (MV-PV), recorrem-se as técnicas de seleção de pares de variáveis. Esse trabalho faz uso dos algoritmos matemáticos da decomposição de valores singulares (SVD), matriz de ganho relativo (RGA) e matriz de ganho relativo para sistemas não quadráticos (NRG); para selecionar os melhores pares e implementar um sistema de controle em duas colunas de destilação do processo produtivo do Etilbenzeno. O processo foi simulado no software Aspen Plus e Dynamics $^{\mathrm{TM}}$, a partir de dados reais de uma unidade. A aplicação das técnicas de seleção de variáveis indicou os melhores pares e verificou o controle de composições por inferência de temperaturas do componente chave pesado, na primeira torre, e chave leve da segunda coluna. O sistema de controle proposto mostrou-se satisfatório quando foram inseridos distúrbios inerentes ao processo, de modo que foram minimizados os efeitos transientes das composições.
\end{abstract}

\section{INTRODUÇÃO}

As unidades industriais investem quando necessitam de uma estrutura de controle robusta e atuante, de modo que o processo opere em uma condição segura, eficaz e com os produtos dentro das especificações. Frequentemente, em processos químicos, nos deparamos com situações nas quais diversos objetivos de controle estão disponíveis, ou seja, diversas variáveis de processos (PV's) devem ser controladas enquanto outras devem ser observadas na condição de distúrbios e variáveis manipuladas (MV's); é o caso de processos multivariáveis (MIMO). Equipamentos como reatores de mistura perfeita (CSTR) e colunas de destilação são exemplos práticos de processos multivariáveis (MORAIS JR, 2014).

O processo produtivo do etilbenzeno (EB) mostrou-se interessante para o presente estudo, porque possui diversas particularidades, dentre as quais se destacam a não linearidade, interatividade, malha de controle acoplada e produtos com alta pureza. A síntese de EB ocorre em dois reatores CSTR em série, a partir da reação do etileno (E) e o benzeno (B); onde também ocorre a formação do composto dietilbenzeno (DEB), originado da reação secundária entre o EB e o B. A saída do segundo reator do processo é corrente de alimentação da 
primeira coluna de destilação multicomponente, sendo seu composto chave leve, o benzeno (>99,9\% molar), reciclado para o primeiro reator do processo. A segunda coluna de destilação tem como alimentação a corrente de base da primeira coluna, fornecendo uma corrente de topo rica em EB (>99,9\% molar) e uma corrente de base rica no DEB (>99,9\% molar), sendo a corrente de base da última coluna usada como corrente de reciclo para o segundo reator. Em colunas de destilação as medições das composições dos produtos de topo e de base não estão disponíveis em tempo real. Sendo assim, o controle das composições em alta pureza por inferência de temperatura motivou o esse trabalho.

\section{FUNDAMENTAÇÃO TEÓRICA}

\subsection{TÉCNICAS DE SELEÇÃO DE PARES DE VARIÁVEIS}

Dentre as principais técnicas de seleção de pares de variáveis para fins de controle se destacam: a decomposição em valores singulares (SVD), matriz de ganho reativo (RGA) e a matriz de ganho reativo para sistemas não quadráticos (NRG).

\subsection{DECOMPOSIÇÃO EM VALORES SINGULARES (SVD)}

A SVD pode ser definida como um algoritmo numérico baseado na teoria de autovalores e autovetores da álgebra linear, com diversas aplicações no âmbito da engenharia. Considere a matriz simétrica $K$, formada pelo ganho em regime estacionário do sistema, de dimensão $\mathbb{R}^{m x m}$. Podemos definir a decomposição de $K$ como,

$$
K_{m x m}=U D P^{T}=\left(u_{1}, \ldots, u_{m}\right)\left(\begin{array}{ccc}
\sigma_{11} & \ldots & \sigma_{1 n} \\
\vdots & \ddots & \vdots \\
\sigma_{m 1} & \cdots & \sigma_{m n}
\end{array}\right)\left(\begin{array}{c}
p_{1} \\
\vdots \\
p_{n}
\end{array}\right)
$$

As matrizes $U$ e $P$ são ortonormais. Os valores singulares $\sigma_{N}$ são os elementos da diagonal principal da matriz $D$; e os autovalores correspondentes à matriz $P$ são $\lambda_{1}, \lambda_{2}, \ldots, \lambda_{n}$,organizados de tal forma que: $\sigma_{1} \geq \sigma_{2} \ldots \geq \sigma_{n}>0$ e $\sigma_{1}=\sqrt{\lambda_{1}}, \sigma_{2}=\sqrt{\lambda_{2}}, \ldots$, $\sigma_{n}=\sqrt{\lambda_{n}}$. Os vetores $u_{1}, u_{1}, \ldots, u_{n}$ e $p_{1}, p_{2}, \ldots, p_{n}$ são vetores singulares à esquerda e à direita, respectivamente. A razão entre o valor singular máximo e o mínimo, denomina-se o número condicional (NC).

$$
N C=\sigma_{11} / \sigma_{m n}
$$

\subsection{MATRIZ DE GANHO RELATIVO (RGA)}

De acordo com MORAIS JR (2014), uma medida de interação não corrompida pelas unidades de variáveis do processo é a RGA, conhecida como a matriz de ganho relativo $\Lambda$. Os elementos de $\Lambda$ são definidos como sendo a razão entre o ganho do sistema em malha aberta e o seu ganho em malha fechada, sendo a RGA definida pela Equação 3. O ganho relativo do sistema, $\lambda_{i j}$ é, definido pela Equação 4. Na equação 4, $P V_{i}$ representa as $i$ variáveis de processo, que neste trabalho, são as temperaturas dos estágios das colunas, e $M V_{\mathrm{j}}$ representa as $j$ variáveis manipuladas. 


$$
\begin{aligned}
& \Lambda=\left[\begin{array}{cccc}
\lambda_{11} & \lambda_{12} & \cdots & \lambda_{1 n} \\
\lambda_{21} & \lambda_{22} & \cdots & \lambda_{2 n} \\
\vdots & \vdots & \vdots & \vdots \\
\lambda_{n 1} & \lambda_{n 2} & \cdots & \lambda_{n n}
\end{array}\right] \\
& \lambda_{i j}=\frac{\left(\partial P V_{i} / \partial M V_{j}\right)_{M V_{k, k \neq j}}}{\left(\partial P V_{i} / \partial M V_{j}\right)_{P V_{k, k \neq i}}}=\frac{\text { GanhoDeMalhaAberta }}{\text { GanhoDeMalhaFechada }}
\end{aligned}
$$

Para sistemas nxn a RGA é calculada a partir da matriz de ganho estacionário, realizando a multiplicação elemento por elemento, $\otimes$, conforme a Equação 5.

$$
\Lambda=K \otimes\left(K^{-1}\right)^{T}
$$

\subsection{MATRIZ DE GANHO RELATIVO NÃO QUADRÁTICO (NRG)}

Chang e Yu (1990) propôs a técnica NRG com o objetivo de medir a interação em um sistema multivariável para sistemas não quadráticos. A NRG é definida como:

$$
\Lambda^{N}=K \otimes\left(K^{\dagger}\right)^{T}
$$

Em que o sobrescrito ${ }^{\dagger}$ representa a pseudo-inversa.

\section{MODELAGEM E SIMULAÇÃO}

O processo produtivo do EB, apresentado no fluxograma da Figura 1, foi simulado em regime estacionário no software Aspen Plus ${ }^{\mathrm{TM}}$, sendo os dados de projeto e condições iniciais obtidas em Luyben (2011). Os reatores operam a volume de $200 \mathrm{~m}^{3}$ e às pressões de 19 e 20 atm, respectivamente. As colunas 1 e 2 (C1 e C2) possuem 21 e 25 estágios, alimentações nos estágios 10 e 15, operando com pressões de 0,1 e 0,3 atm, respectivamente. O modelo termodinâmico empregado para os cálculos das propriedades físicas foi de Chao-Seader. Os equipamentos do processo foram dimensionados para operar em regime transiente.

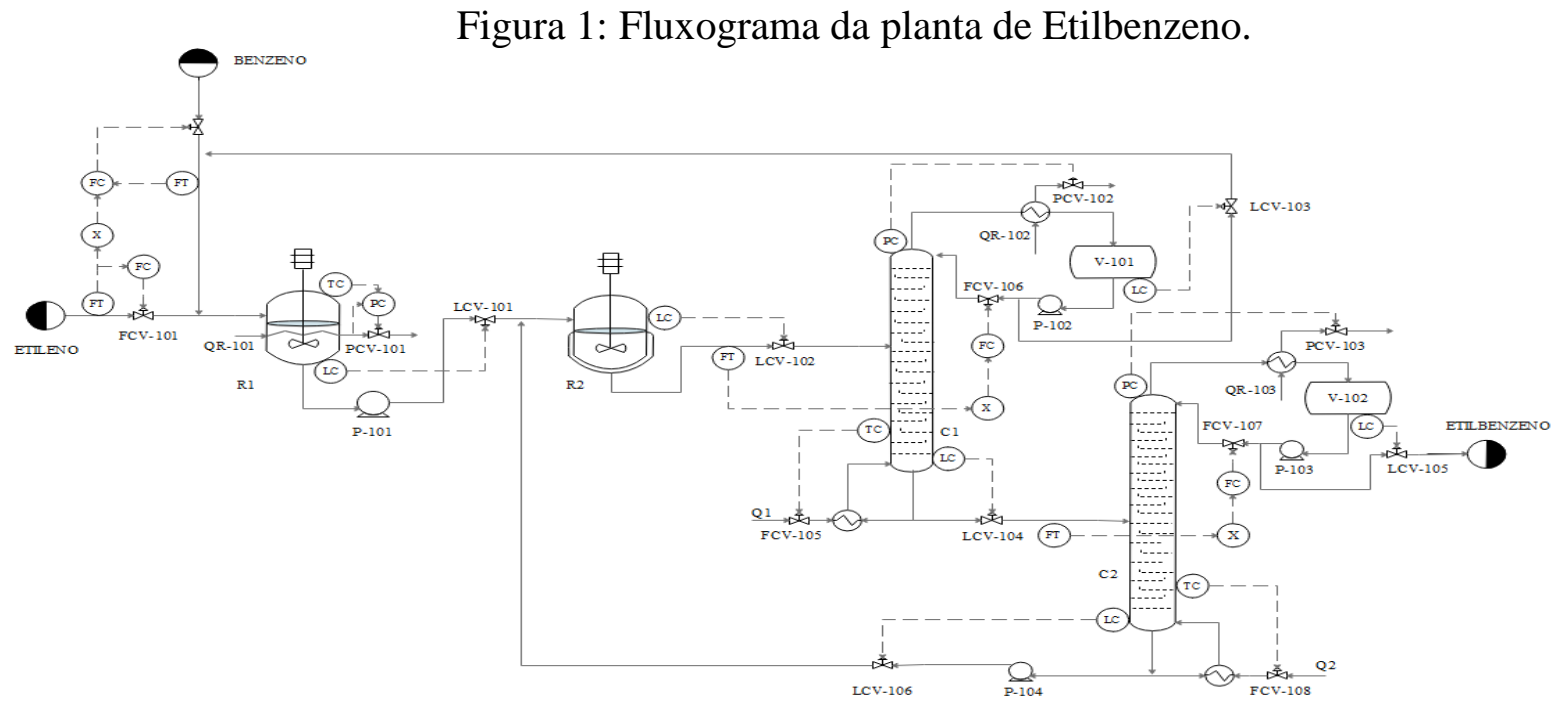


Os gráficos das Figuras 2.a e 2.b apresentam os perfis de composição ao longo dos estágios de $\mathrm{C} 1$ e $\mathrm{C} 2$. Verifica-se de acordo com a figura 2.a que em $\mathrm{C} 1$ o benzeno, $x_{\mathrm{B}}$, sai em alta pureza no estágio 1 (topo). De acordo com a figura 2.b, o Etilbenzeno e o Dietilbenzeno, $x_{\mathrm{EB}}$ e $x_{\mathrm{DEB}}$, estão em alta pureza nos estágios 1 e 25 (topo e base) de $\mathrm{C} 2$, respectivamente.

Figura 2. Perfis de composição molar nas colunas de destilação C1 e C2.

\section{2.a Coluna $\mathrm{C} 1$.}

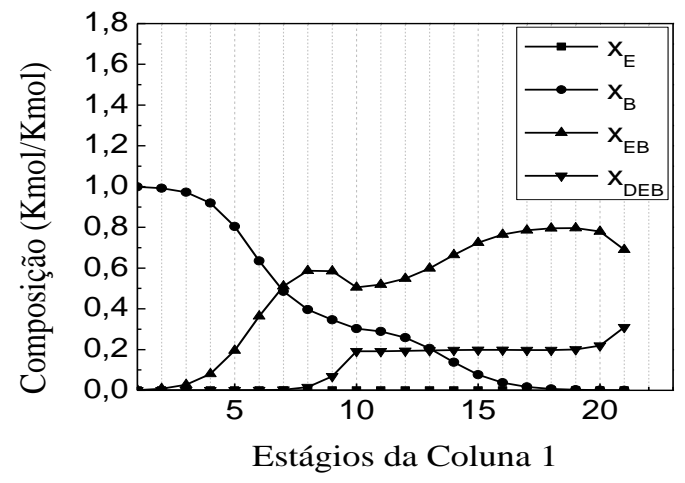

2.b Coluna $\mathrm{C} 2$.

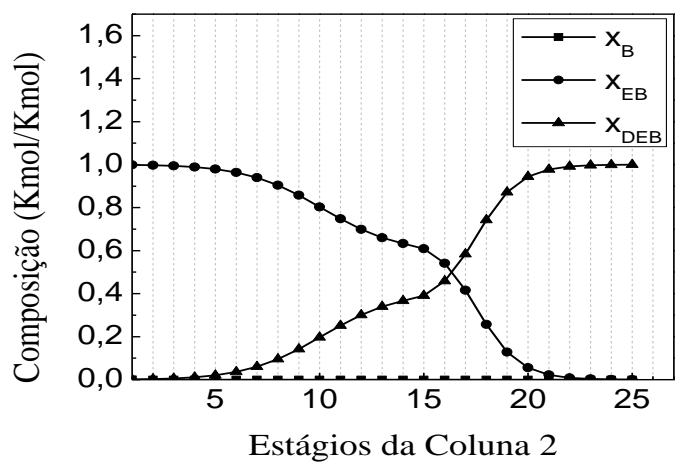

\section{RESULTADOS}

Para obtenção das matrizes de ganho estacionário foram efetuadas perturbações com amplitude de 5\% nas variáveis: $\mathrm{D}_{1}, \mathrm{R}_{1}, \mathrm{RR}_{1}$ e $\mathrm{Q}_{1}(\mathrm{C} 1)$; e $\mathrm{D}_{2}, \mathrm{R}_{2}, \mathrm{RR}_{2}$ e $\mathrm{Q}_{2}(\mathrm{C} 2)$. Em que, $\mathrm{D}, \mathrm{R}$, RR e Q refere-se a corrente de destilado, refluxo, razão de refluxo e carga térmica, respectivamente. $O$ índice 1 é referente a primeira coluna e o 2 a segunda coluna.

Os algoritmos da SVD, RGA e NRG foram implementados no software Matlab®. De acordo com a tabela 1, o par de MV que apresenta menor NC é $\mathrm{D}_{1}-\mathrm{R}_{1}$. No entanto, essas variáveis são utilizadas para controle de nível do vaso de refluxo e vazão de alimentação de $\mathrm{C} 1$, respectivamente. Sendo assim, o próximo par com menor $\mathrm{NC}$ é $\mathrm{RR}_{1}-\mathrm{Q}_{1}$, selecionado para controle de temperatura em $\mathrm{C}_{1}$. Para $\mathrm{C} 2$ os resultados da tabela 2 indicam que os três primeiros pares são os que têm menores NC, entretanto essas variáveis já são empregadas no controle de outras variáveis. Assim, o par selecionado foi $\mathrm{RR}_{2}$ e $\mathrm{Q}_{2}$.

Tabela 1 - Número Condicional $\mathrm{C}_{1}$

\begin{tabular}{|c|c|}
\hline Pares & NC \\
\hline $\mathrm{D}_{1}$ e R & 1,856 \\
\hline $\mathrm{RR}_{1}$ e $\mathrm{Q}_{1}$ & 18,809 \\
\hline $\mathrm{D}_{1}$ e $\mathrm{Q}_{1}$ & 118,798 \\
\hline $\mathrm{D}_{1}$ e $\mathrm{RR}_{1}$ & 489,080 \\
\hline $\mathrm{R}_{1}$ e $\mathrm{Q}_{1}$ & 4500,0584 \\
\hline
\end{tabular}

Tabela 2 - Número condicional $\mathrm{C}_{2}$

\begin{tabular}{|c|c|}
\hline Pares & $\mathrm{NC}$ \\
\hline $\mathrm{D}_{2}$ e $\mathrm{R}_{2}$ & 2,257 \\
\hline $\mathrm{B}_{2}$ e $\mathrm{R}_{2}$ & 9,217 \\
\hline $\mathrm{B}_{2} \mathrm{e} \mathrm{Q}_{2}$ & 19,476 \\
\hline $\mathrm{RR}_{2}$ e $\mathrm{Q}_{2}$ & 20,330 \\
\hline $\mathrm{D}_{2}$ e $\mathrm{Q}_{2}$ & 94,667 \\
\hline
\end{tabular}

De acordo com as figuras 3 e 4, os estágios que apresentaram maior sensibilidade foram 13 e 16 para C1, e 17 e 21 para C2. Pode-se, então, recorrer a RGA, para confirmar ou não a seleção do par PV-MV mais adequado. A RGA confirmou os resultados da SVD, indicando que a carga térmica $\left(\mathrm{Q}_{1}\right)$ deve ser empregada para controlar a temperatura do estágio $13\left(\mathrm{~T}_{13}\right)$ de $\mathrm{C} 1$ e a carga térmica $\left(\mathrm{Q}_{2}\right)$ deve ser empregada para controlar a temperatura do estágio $17\left(\mathrm{~T}_{17}\right)$ de $\mathrm{C} 2$. Esses resultados são apresentados nas tabelas 3 e 4, tomando como 
base o fato que ganhos relativos próximo de uma unidade indicam que há interação entre as malhas e o controle é viável, enquanto valores negativos indicam o contrário.

Figura 3 - Matriz de sensibilidade para C1. Figura 4 - Matriz de sensibilidade para C2.
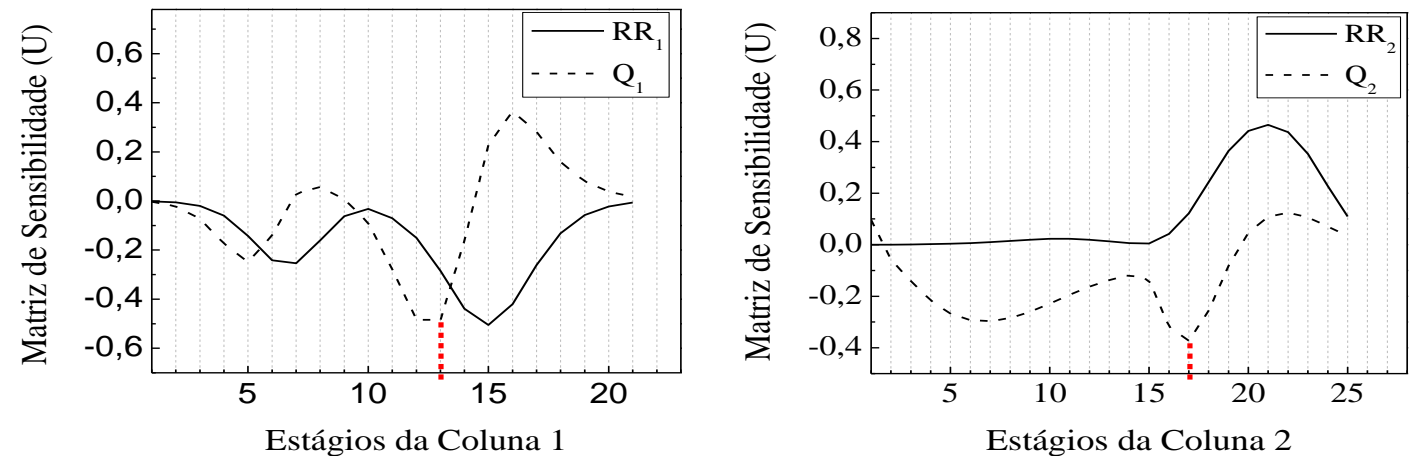

Os resultados da NRG foram análogos aos obtidos com a SVD e RGA, indicando os pares ideais $T_{13}-Q_{1}(C 1)$ e $T_{17}-Q_{2}(C 2)$, conforme apresentam, respectivamente, os gráficos das figuras 5 e 6 . Com esses resultados foram implementados e sintonizados os controladores de temperaturas TC_13 e TC_17, indicados na figura 1. Para verificar o desempenho dos controladores foram efetuadas perturbações de $+5 \%$ (no tempo de $10 \mathrm{~h}$ ) e $-5 \%$ (no tempo de 30h), conforme observado nos gráficos das Figuras 7 e 8. Para essas mesmas perturbações, verifica-se que os controladores de temperatura minimizaram a sobre-elevação percentual (overshoot) das composições de $\mathrm{x}_{\mathrm{EB}}$ (base de C1), $\mathrm{x}_{\mathrm{B}}$ (topo de C1), $\mathrm{x}_{\mathrm{EB}}$ (topo de C2), $\mathrm{x}_{\mathrm{DEB}}$ (topo de C2) em comparação com a condição de malha aberta (MA), conforme apresentam os gráficos das figuras $9,10,11$ e 12 , respectivamente.

Tabela 3 - Resultado da RGA para $\mathrm{C}_{1}$

\begin{tabular}{|c|c|c|}
\hline Pares & $\mathrm{RR}_{1}$ & $\mathrm{Q}_{1}$ \\
\hline $\mathrm{T}_{13}$ & $-0,1825$ & 1,1825 \\
\hline $\mathrm{T}_{17}$ & 1,1825 & $-0,1825$ \\
\hline
\end{tabular}

Figura 5 - NRG para o par $\mathrm{RR}_{1}$ e $\mathrm{Q}_{1}$.

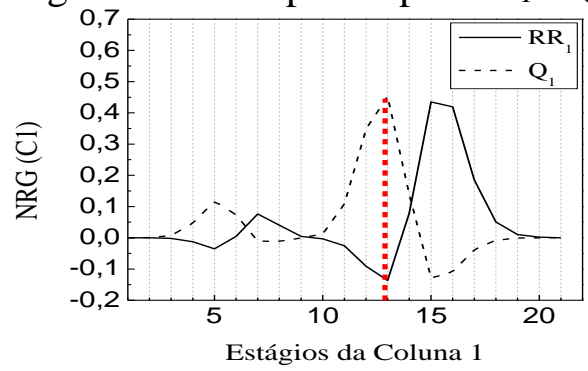

Figura 7 - Comportamento de $\mathrm{T}_{13}$ em C1.

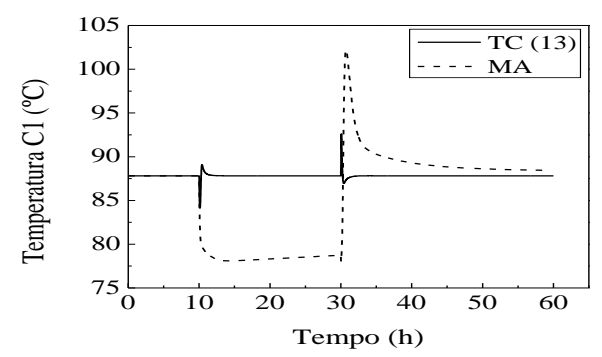

Tabela 4 - Resultado da RGA para $\mathrm{C}_{2}$

\begin{tabular}{|c|c|c|}
\hline Pares & $\mathrm{RR}_{2}$ & $\mathrm{Q}_{2}$ \\
\hline $\mathrm{T}_{17}$ & $-0,02581$ & 1,02581 \\
\hline $\mathrm{T}_{21}$ & 1,02581 & $-0,02581$ \\
\hline
\end{tabular}

Figura 6 - NRG para o par $\mathrm{RR}_{2}$ e $\mathrm{Q}_{2}$.

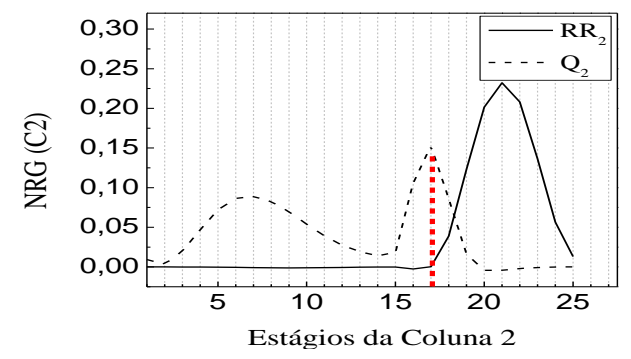

Figura 8 - Comportamento de $\mathrm{T}_{17}$ em C2.

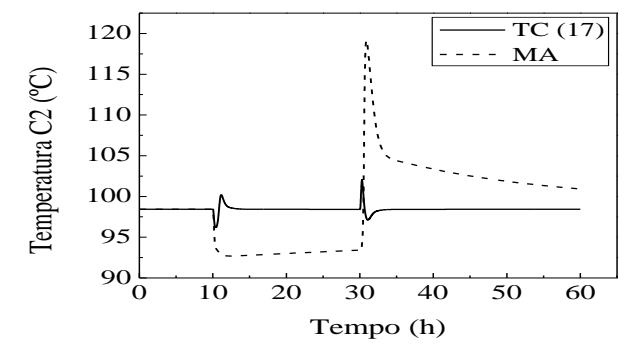




\section{Congresso Brasileiro de Engenharia Química em Iniciação Científica UFSCar - São Carlos - SP 16 a 19 de Julho de 2017}

Figura 9 - Comportamento transiente da composição de $\mathrm{EB}\left(x_{\mathrm{EB}}\right)$ na base de $\mathrm{C} 1$.

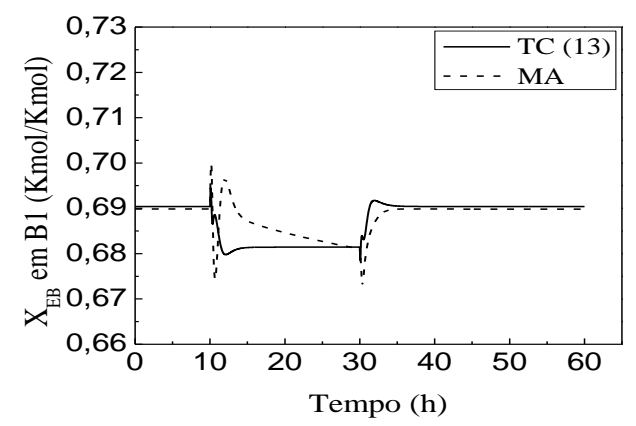

Figura 11 - Comportamento transiente da composição de EB $\left(x_{\mathrm{EB}}\right)$ no destilado de $\mathrm{C} 2$.

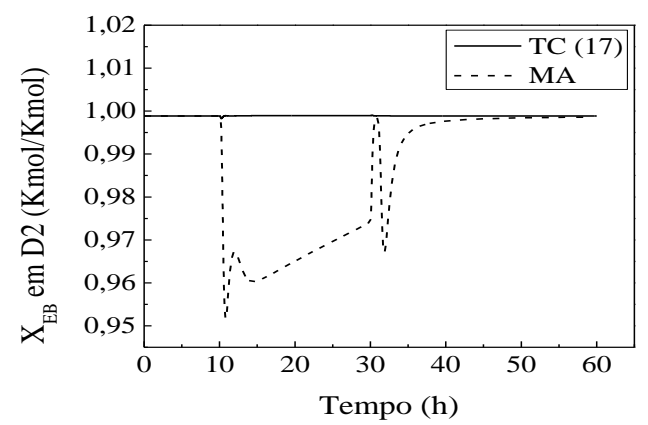

Figura 10 - Comportamento transiente da composição de $\mathrm{B}\left(x_{\mathrm{B}}\right)$ no destilado de $\mathrm{C} 1$.

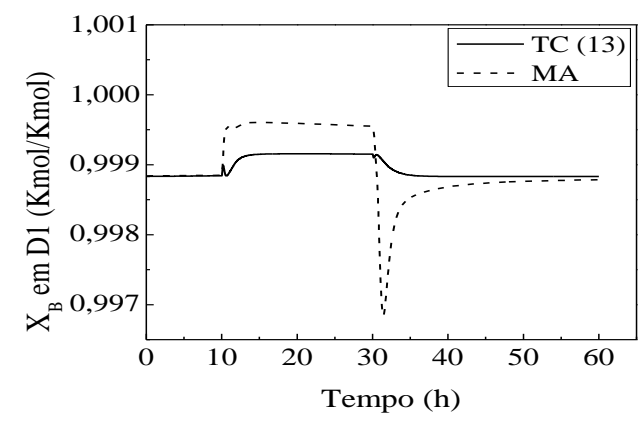

Figura 12 - Comportamento transiente da composição de DEB ( $\left.x_{\mathrm{DEB}}\right)$ na base de $\mathrm{C} 2$.

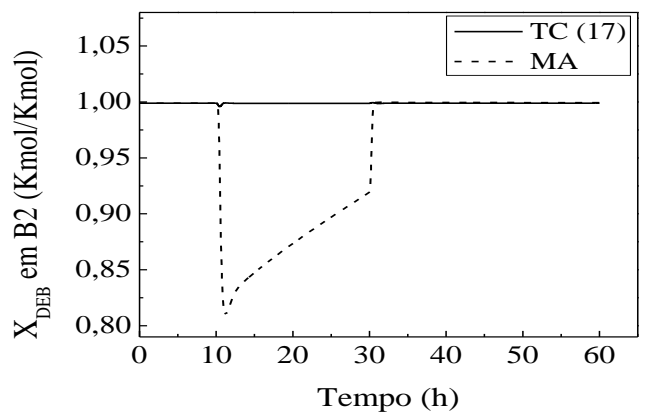

\section{CONCLUSÕES}

O uso das técnicas SVD, RGA e NRG mostraram-se eficientes na seleção de estrutura de controle de temperatura para as colunas de destilação do processo do Etilbenzeno. De acordo com esse trabalho, verificou-se que as aplicações do controle singular por inferência de temperaturas mantiveram as respostas das composições com alta pureza, dentro de patamares aceitáveis para o processo. Assim, verificou-se de fato que o controle foi atuante para as composições $\mathrm{X}_{\mathrm{EB}}$ (topo de C2), $\mathrm{X}_{\mathrm{DEB}}$ (topo de C2), Figuras 11 e 12, haja vista, que alterações na vazão de alimentação de $\mathrm{C} 1$ e $\mathrm{C} 2$, Figuras 7 e 8, proporcionam grandes distúrbios na temperatura dos estágios de maior sensibilidade, $\mathrm{T}_{13}$ (C1) e $\mathrm{T}_{17}$ (C2), e consequentemente nas composições de topo e de base.

\section{REFERÊNCIAS}

CHANG, J. W., \& Yu, C.-C. (1990). The relative gain for non-square multivariable systems. Computers and Chemical Engineering, 45(4), 1309-1323.

LUYBEN, W. L. 2012. Distillation Design and Control Usign Aspen Simulation. New Jersey. Ed: John Wiley \& Sons, 2nd.

MORAIS JR, A. A., 2014. Seleção ótima de entradas no estudo de inferência e controle de composições em um processo de destilação industrial de alta pureza. Congresso Nacional de Matemática Aplicada à Indústria-CNMAI, Caldas Novas - GO. 